\title{
Nuestros cuerpos, nuestra tierra: La política de renovación, reestructuración y (re)evolución
}

\section{IRENe DiAmond*}

\section{Resumen}

En este texto de principios de los años noventa Irene Diamond ofrece una critica ecofeminista al dominio del conocimiento técnico y a la narrativa acerca del progreso características de la ilustración Si bien Diamond incide en el daño que estos procesos pueden generar, más si cabe que "la devastación ya conocida del militarismo violento", también ofrece una contundente respuesta a favor de un futuro ecológico, democrático e igualitario a partir de la lucha de las mujeres no occidentales en pos de su propia liberación así como de la liberación de la tierra; una lucha que Diamond entronca con varias tendencias del feminismo contemporáneo en general, y del ecofeminismo en particular: la defensa de la interseccionalidad, la revalorización del conocimiento situado y activista, la articulación de una agenda de emancipación holística y libertaria que cuestiona el potencial emancipador del estado, y la puesta en práctica de dos eslóganes que han venido a definir las luchas feministas y ecologistas en los últimos tiempos: lo personal es político ( $y$ académico) y piensa globalmente, actúa localmente.

\section{Palabras clave}

Feminismo; ecologismo; ecofeminismo; Ilustración; interseccionalidad.

\section{TitLe}

Our Politics, Our Earth: The Politics of Renewal

\section{Abstract}

In this text from the early 1990s, Irene Diamond offers an ecofeminist critique of the domination of tecnocratic knowledge and the narrative of progess that characterised the Enligthenment. While Diamond insists on the harm that such processes can generate, even more so than the devastation created by violent militarism, she also offers a strong answer in favor of an ecological, democratic and egalitarian future by drawing on the intricate struggle of non-western women for their liberation as well as the liberation of the earth; a struggle that Diamond relates to various tendencies within contemporary feminism in general, and ecofeminism in particular: the defense of intersectionality, the valorisaion of activist and situated knowledge, the articulation of a holistic emancipatory agenda of a libertarian nature, that questions the emancipatory potential of the state and the performance of two slogans which have come to define ecologists and feminists' struggles: the personal is political (and academic), and think global, act local.

\section{KEYWORDS}

Feminism; ecologism; ecofeminism; Enlightenment; intersectionality.

\section{*Irene} DIAMOND,

Profesora asociada en la facultad de ciencia política de la Universidad de Oregon, Estados Unidos, donde da clase de ciencia política y estudios de género. Entre sus publicaciones más importantes destacan los volúmenes Feminism and Foucault: Reflections on Resistance, coeditado con Lee Quinby, y Reweaving the World: The Emergence of Ecofeminism, también coeditado junto con Gloria Feman Orenstein.

\section{Todos los} derechos de este texto pertenecen a Beacon Press e Irene Diamond. Reproducido con permiso de la editorial, Beacon Press. Capítulo original: Diamond, Irene, "Our Politics, Our Earth: The Politics of Renewal", en Fertile Ground, Ed. Beacon Press, Boston, 1994, ps. 202. Copyright (C) 1994 de Irene Diamond.

Traducido por Lucrecia RUBIO GRUNDELL, estudiante de doctorado en el Instituto Europeo de Florencia

DOI: $10.15366 /$ relacionesinternacio nales2017.34.004 
U

na de las crueles paradojas a la que nos enfrentamos hoy en día es que en esta era de transición política pos-Guerra Fría, nuestras intervenciones con las fuerzas de la creación -el esfuerzo insistente por mejorar la vida e imponer orden en sus desviaciones e irregularidades- bien puede traer más daño y dolor que la devastación ya conocida del militarismo violento. Esta posibilidad se vuelve especialmente vívida cuando notamos la habilidad de hoy para aislar genes vivientes y transferirlos de un organismo a otro. En un ambiente cultural cautivado por el conocimiento técnico, no resulta sorprendente que tantos de quienes se preocupan por la degradación medioambiental identifiquen los avances en biotecnología, rayos láser, anticonceptivos u ordenadores como "la" solución para los problemas medioambientales y de salud. La fe en el progreso tecnológico nos hace perder conciencia, convenientemente, del modo en que ésta tecnología es cómplice de las fuerzas que han producido nuestro contemporáneo dilema.

Ante esta tendencia dominante que evita los valores y es ciega frente a lo que se pierde cuando insistimos en ser amos de la naturaleza, mantener la esperanza por un futuro ecológico, democrático e igualitario es una lucha constante. La narrativa humanitarista estándar de progreso trae esperanza a las masas hambrientas, oprimidas e ignorantes que son milagrosamente elevadas por la beneficencia, y sobre todo la tecnología, de los donantes occidentales. No obstante, por mi propia experiencia, me ha resultado más difícil mantener la esperanza entre los privilegios de ser ciudadana de una nación occidental imperialista como Estados Unidos. Por supuesto, Estados Unidos tiene partidos verdes e instituciones de base alterativas que luchan por encarnar valores y prácticas más igualitarias y sostenibles. Sin embargo, constantemente encuentro mi espíritu y mis visiones utópicas atenuadas aquí en vistas de la pobreza y exclusión social en medio de la abundancia, y soy dolorosamente consciente de la propagación del consumismo. A menudo no estoy nada segura de cómo actuar. Aquí debo vivir, de algún modo, con el conocimiento de que la avaricia y el amor por el lujo de nuestra nación son responsables del despojo a la Tierra y de causar estragos en aquellas culturas que no se organizan alrededor de la búsqueda de la abundancia material. Mi sentido de las monstruosas prácticas de colonización de Estados Unidos y otras naciones occidentales es a veces tan abrumador que no estoy segura de cómo puedo apoyar la experimentación con la resistencia que está siendo articulada de manera tan brillante y creativa en el seno de países que sufren la miseria y dislocación forjadas por el desarrollo. En efecto, al regresar de un viaje a la India, me encontré cayendo en una grave depresión. Sólo cuando me comunique - vía ordenador - con una amiga estadounidense, también regresada recientemente, y que también estaba experimentando una depresión, fui capaz de establecer el vínculo entre mi depresión y mi dilema en torno a una ética apropiada para aquellas de nosotras que vivimos en el mundo occidental.

Mis experiencias en India me enseñaron que muchos de los movimientos de resistencia más interesantes estaban lejos de las capitales y de las más prestigiosas instituciones de educación superior y producción de conocimiento. La perspectiva crítica de las portavoces del tercer mundo en el nuevo aula global de las principales ciudades de occidente a menudo se acerca más a la cosmovisión eurocéntrica que está siendo atacada, en vez de a las diversas visiones de sus hermanas y hermanos que no han tenido el acceso privilegiado a esa educación occidental. Aunque no tengo ninguna ilusión de que yo pueda trascender las limitaciones de mis propios privilegios, mi preocupación por el modo en el que la producción de conocimiento 
eurocéntrico está eliminado modos alternativos de conocer y hacer me ha llevado a prestar particular atención a las mujeres campesinas de la India.

En el tumulto del fin de la Guerra Fría, la reafirmación del nacionalismo violento ha dado lugar a cierto escepticismo en torno a las soluciones locales y a pequeña escala frente a los problemas generados por enormes estados nación y corporaciones transnacionales. Yo creo, sin embargo, que tenemos que prestar más atención a prácticas alternativas vinculadas a lugares y regiones particulares. Aunque el localismo no ofrece ningún tipo de garantías, los efectos del desarrollo del mercado global indican inequívocamente que es mucho más probable que las prácticas igualitarias, inclusivas y ecológicamente sostenibles deriven del cuidado y el conocimiento de lugares locales.

Muchas mujeres campesinas en India, que rara vez tienen acceso a los privilegios educacionales y de clase de sus hermanas más elitistas, desafían de manera fundamental las asunciones de las expertas del "primer mundo" y sus aliadas del Sur. Los expertos del Norte típicamente promueven una "política de restructuración" que privilegia la avaricia, la velocidad, la dependencia, y el poder del conocimiento burocrático. Por el contrario, muchas mujeres Indias en niveles populares, especialmente aquellas mujeres tribales y campesinas que siguen manteniendo fuertes vínculos con la tierra, abogan por una "política de renovación" que fomenta la generosidad, la reparación que a menudo viene con el simple paso del tiempo, la auto subsistencia local, y el poder de la solidaridad comunitaria y la ayuda mutua. Las mujeres populares del subcontinente indio buscan mantener y/o reconstruir la diversidad y seguridad de lo común y las formas de vida locales, desafiando los proyectos de desarrollo y cercamiento de lo común por el mercado. Como sugieren los autores anónimos del número especial sobre "la debacle de la cumbre del clima" de 1992 en The Ecologist, que titularon provocativamente "¿El futuro común de quién?", el mercado del mundo desarrollado "implica un impulso intransigente hacia una estructura global única equipada con mecanismos de vigilancia global y de conversión global de recursos que alimentan un avance material ilimitado"1. Desestimando las relaciones y los valores personales que derivan del estar enraizada en lugares particulares, la política de la reestructuración, sin duda la respuesta dominante al actual predicamento ecológico, sólo puede imaginarse un "futuro común" asegurado a través de la administración global y de una mayor vigilancia tanto de las personas como del mundo viviente.

Una alternativa simple, aunque profunda, a esta administración y esta vigilancia -que se traduce fácilmente en una mayor vigilancia de los cuerpos de las mujeres- se encuentra inscrita en el eslogan exhortatorio de Shri Mukti Sangarsh Calwal, el Movimiento de Liberación de Mujeres de las aldeas campesinas del distrito de Sangli, del Estado de Maharashtra: "Tierra Verde, Poder de las Mujeres, Liberación Humana". Cuando escuché por primera vez este alegato en el otoño de 1991, estaba emocionada. Parecía que en Estados Unidos, donde las identidades están fracturadas y el pensamiento compartimentado, ya no teníamos acceso a una visión tan humanista. En un nivel más grandioso, el eslogan sugiere un camino fuera del dilema de la Ilustración - la maestría técnica sobre el mundo natural también encarcela la capacidad humana para el placer polimorfo y las relaciones igualitarias. La visión de Shri

$1 \quad$ VVAA, "Global Management" en The Ecologist, vol. 22, n 3, 1992, p. 180. 
Mukti de la libertad como algo dependiente del bienestar de la Tierra y del reconocimiento de las mujeres como personas plenas se mueve audazmente en la dirección de eliminar la oposición entre lo humano y lo animal, hombre y mujer, conocimiento y placer que enmarcan desde hace mucho en el entendimiento occidental la condición humana.

Empoderadas por tales expresiones contemporáneas de verdades no eurocéntricas, quizás nosotras en occidente podemos empezar a imaginar más fácilmente alternativas a la lógica del control y la dominación, alternativas que no se apresuren a contener y ordenar el desorden vital, o a insistir en la superioridad epistemológica de lo transcendente, lo masculino y lo visual. Esta recultivación de formas de conocer que han sido empequeñecidas, cuando no erradicadas por la construcción de la verdad por parte de la Ilustración y el cercamiento de la tierra y los pueblos, crea la posibilidad de placeres expandidos y de reparar la separación entre sujeto y objeto que ha dado lugar a la pesadilla de un mundo totalmente irradiado.

No es accidental que encontremos esta esperanza de libertad y cambio fundada y basada en la Tierra en un entorno cultural y económico donde la sabiduría ecológica se deriva de las prácticas cotidianas de las comunidades rurales. En occidente, nuestra consciencia sobre la interconexión global tiende a ser abstracta y distante, como tan bien simboliza ese adorado icono medioambiental posmoderno, la foto del mundo entero producida por la NASA. Para muchos en Estados Unidos, esta fotografía de alta tecnología evoca poderosamente la belleza y la fragilidad de la Tierra. No obstante, esa imagen unívoca, oculta simultáneamente los conocimientos locales y la realidades de poder y dominación globales ${ }^{2}$. Las amenazas al bienestar y a la integridad de la biosfera por el imperativo de crecer a cualquier precio, con sus esquemas homogeneizadores de agricultura industrializada, mercados centralizados y valores de consumo, necesita nuevas imágenes, visiones y formas de resistencia política.

Para las mujeres rurales del Sur que se preocupan por el agua, el combustible, la comida y el forraje para la subsistencia, las oportunidades del mercado global representan típicamente una intrusión en su modo de vida. Su destino está íntimamente entrelazado con el bienestar de la Tierra, pero el hogar que estas mujeres quieren y luchan por proteger no es la Tierra entera en abstracto. Al contrario, hogar y Tierra son las tierras, bosques y ríos particulares que conocen y cuidan personalmente en sus vidas cotidianas. Para estas mujeres las esperanzas de liberación no pueden satisfacerse con reivindicaciones de un único hogar terrenal; no caen en el mito de que cuando nos enfrentamos a temas de devastación ecológica de algún modo somos todos responsables. Tampoco se imaginan que pueda haber formas políticas, culturales o económicas de renovación que no tengan en cuenta la sabiduría tradicional de las mujeres.

Por muy locales que sean las preocupaciones de estas mujeres, sin embargo, también construyen alianzas. Shri Mukti Sangarsh Calwal, por ejemplo, ha trabajado con otras organizaciones campesinas en la lucha contra la sequía en Sangali y otros distritos cercanos, así como junto con organizaciones campesinas más grandes y lideradas por hombres en todo el estado - "Shetkari Sanghatana". Esta organización de masa, liderada por el líder carismático

\footnotetext{
2 Para una crítica de la imagen total de la Tierra, véase GARB, Jaakov Jerome, "Perspective or escape? Ecofeminist Musings on Contemporary Earth Imaginery" en DIAMOND, Irene, ORENSTEIN, Gloria Feman (eds.), Reweaving the World: The Emergence of Ecofeminism, Sierra Club, San Francisco, 1990, ps. 264-278.
} 
Sharad Joshi, con una amplia base de seguidores en la India, es conocida principalmente por su tabla de precios remunerativos para los campesinos, aunque en años recientes Shetkari Sanghatana también ha comenzado a agitar cuestiones como la agricultura natural, la igualdad de derechos de las mujeres con respecto a la propiedad, y la elección de paneles compuestos sólo por mujeres para los órganos de gobierno locales, los gram panchayat ${ }^{3}$. Las severas sequías que afectan esta región sumadas a las prácticas de la agricultura industrializada han llevado tanto a mujeres como a hombres a reevaluar las prácticas de agricultura tradicionales que no dependían del uso de inputs externos que debían comprarse en el mercado. Muchas de estas prácticas más tradicionales estaban vinculadas típicamente a festivales religiosos que honraban y celebraban los ciclos de renovación de la Tierra. Shri Mutli Sangarsh, como muchos otros grupos de base en la India - desde mujeres pescadoras en Kerala a mujeres tabacaleras en Nipani y activistas tribales en Maharashtra - han comenzado así una discusión acerca del desarrollo alternativo o contra desarrollo desde la perspectiva de las mujeres ${ }^{4}$.

Muchas activistas ahora afirman que la Revolución Verde en la agricultura no sólo hizo a la comunidad campesina más dependiente de peligrosos pesticidas, fertilizantes químicos, y semillas híbridas que debían comprarse en mercados controlados por el estado, también privó a las mujeres de su poder de decisión a la vez que incrementó su trabajo. Esta degradación de los patrones tradicionales sostenibles ha llevado a algunas activistas a llamar a la esperanza del desarrollo -recién reconfigurada como la esperanza del desarrollo sostenible- "la guerra por otros medios" ${ }^{\prime \prime}$. Se argumenta que en las prácticas agrícolas tradicionales las mujeres mantenían las semillas para plantar, a menudo decidiendo dónde y cuándo hacerlo. En algunas áreas controlaban incluso la compraventa de ganado y ovejas. Hoy en día, sin embargo, las mujeres han sido desplazadas del sistema económico centralizado, mientras que la sequía ha aumentado la carga que deben soportar. El empoderamiento económico y político de las mujeres reside, no en hacer demandas al estado, sino en luchar a nivel local para generar cambios en los niveles mitológicos, de la aldea y el hogar. En vez de insistir en el acceso a los degradantes programas de bienestar del estado, están recurriendo a su ingenio colectivo para restaurar medios sostenibles de supervivencia.

La reciente campaña de Laxmi Multi en la que han participado activistas en la zona rural de Maharashtra ejemplifica el modo en el que las luchas ecológicas contemporáneas a menudo participan de mitos y símbolos tradicionales. Esta campaña por la redistribución de la tierra utiliza imagineria de la deidad hindú -imágenes mayormente despreciadas por las feministas urbanas- para visibilizar sus esfuerzos. La campaña por los derechos de las mujeres sobre la tierra llama a los hombres a poner una porción de su tierra a nombre de sus mujeres. Esta lucha por la liberación, "mukti", ha sido llamada Laxmi Mukti siguiendo a Laxmi, la Diosa Hindú de la fortuna. Se han iniciado esfuerzos por recolectar información y recursos para poder restaurar un antiguo templo Sita en una de las aldeas, que es una fortaleza de

GURU, Gopal, "Latmi Mukti" en Economic and Political Weekly, Julio, 1992; OMVEDT, Gail, "Latmi Mukti" en Hitavada, 27 de Diciembre, 1992.

Véase SEN, Ilina, (ed.), A Space Within the Struggle: Women's Participation in People's Movements, Kali for Women, Nueva Deli, 1990; OMVEDT, Gail, The Awakening of Women's Power: The Rural Women's Movement in India, manuscrito no publicado, 1992.

Para una crítica del report Brundtland y el desarrollo sostenible véase VISVANATAN, Shiv, "Mrs. Brudtland's disenchanted Cosmos" en Alternatives, Social Transformation and Humane Governance, vol. 16, no 3, 1991, ps. 377-84. 
Shetkari Sangathana. Los campos experimentales donde las técnicas de agricultura naturales que no dependen de inputs externos están siendo investigadas se llaman los campos de Sita. La Diosa mitológica Sita que, en tanto que la mujer desterrada del Dios Ram, prosperó a pesar de un doloroso exilio, simboliza la persistente fuerza de las mujeres dentro de los confines del patriarcado. Las mujeres involucradas en las campañas Laxmi Mukti de hecho, están revitalizando los fórums comunitarios y aldeanos para lidiar con la supervivencia cotidiana. En efecto, están creando un modo de hacer política democrática que empodera a la gente corriente y a la vez lucha contra la mentalidad gerencial de las expertas en desarrollo, las planificadoras familiares, y el estado.

Las diversas, aunque en muchas maneras similares, luchas de las mujeres alrededor del planeta son recuerdos tangibles de que las mujeres a menudo son líderes cruciales en el mantenimiento de las comunidades y los ecosistemas locales. Mientras que los administradores globales entienden el medioambiente típicamente en términos de lo que está ocurriendo en la economía, para las mujeres activistas en los niveles de base el medioambiente es aquello que rodea sus hogares, aldeas, y comunidades particulares ${ }^{6}$. Al mismo tiempo, sus variadas resistencias no son meros esfuerzos para preservar el status quo, pues típicamente desafían prácticas y procedimientos establecidos que tratan a la Tierra como un mero recurso para uso humano. Mujeres como Lois Gibbs en relación a las toxinas químicas de Love Canal, Rachel Jones Bagby entre la devastación urbana de Filadelfia, Wangari Maathai respecto a la deforestación y las tierras devastadas de Kenia, y Medha Patkar en los conflictos entre granjeros, tribales, defensores del medioambiente y expertos en desarrollo en torno a la presa Narmada en la India, están contribuyendo a crear nuevas alianzas, a desarrollar nuevas estrategias, y a revitalizar la esperanza de que existan comunidades diversas y sostenibles, cuando toda esperanza parece haberse desvanecido?.

Poniendo en primer plano la interdependencia del bienestar humano y planetario, las luchas de estas mujeres nos permiten imaginar el florecimiento de nuevas formas de aventura cuyo placer deriva de no de la emoción de seres aislados participando en una conquista, sino del regocijo de una participación política comprometida, el juego de la creatividad artística, y la maravilla de dejar que los misterios sean desvelados. En "Una Utopía Feminista", Teresa Santa Clara Gomes, diputada del partido socialista del parlamento portugués, pondera esta búsqueda contemporánea de una nueva sabiduría, "lo que queda para las sociedades es la utopía volver a la sabiduría fundamental, el arte de asombrarse cuando una se enfrenta al misterio inmenso, desconocido, insondable que establece el ritmo de cambio en el universo"8. Mi esperanza es que con la transformación de este tradicional deseo masculino de aventura, la emoción de la conquista sexual sea reemplazada por el asombro de la conexión sensorial en todas sus expresiones, de tal manera que se realice la política democrática y evolucione un nuevo y dinámico sentido de paz.

\footnotetext{
$6 \quad$ Mi discusión en este capítulo sobre los gerentes globales y sus esfuerzos por cercar más si cabe lo común saca mucho del número especial de The Ecologist, vol. 22, no. 3, 1992, titulado "Whose Common Future?".

GIBS, Lois, como se lo contó a LEVINE, Murray, Love Canal: My Story, State University Press of New York, Albany, 1982; BAGBY, Rachel, "Daughters of Growing Things" en DIAMOND, Irene, ORENSTEIN, Gloria Feman (eds.), Reweaving the World... op. cit.; MAATHAI, Wangari, The Greenbeld Movement, Environmental Liason Centre International, Nairobi, 1988; entrevista con PATKAR, Medha en India International Centre Quarterly 1990, vol. 19, no 1-2, 1992, ps. 273-99.
}

$8 \quad$ GOMES, Teresa Santa Clara, "A Feminist Utopia" en Terra Femina, p. 89. 
La paz, en su sentido más amplio - el fin del sistema global de militarismo y estados nación que son la expresión institucionalizada de la violencia, la no violencia como una máxima de la vida cotidiana, y el respeto por el poder del amor y la compasión- es una piedra angular del ecofeminismo y la política verde contemporánea. El campamento de mujeres militante, y aún así lleno de amor, en la instalación militar de Greenham Common en Inglaterra, se mantiene como uno de los símbolos contemporáneos de políticas por la paz más importantes. Las nuevas políticas de paz y no violencia no idealizan la pasividad. Tampoco aspiran a un equilibrio estático, perpetuo. Ecofeministas y verdes alrededor del mundo, desde la tardía Petra Kelly de Alemania a las activistas por la paz Corinne Kumar-D'souza de Bangalore, India, y el crítico marxista y activista social Gail Omvedt de Kasegaon en la rural Maharashtra, se toman en serio la condena de Ghandi a la sumisión pasiva frente a la injusticia. Haciéndolo, enfatizan los vínculos fundamentales entre la cordura ecológica, justicia social, y la erradicación del militarismo, vínculos que no estaban tan bien articulados con anterioridad por individuos y movimientos protestando las injusticias sociales y las grandes guerras de este siglo.

Pero como indican las chocarrerías que también estuvieron presentes en el bloqueo de Greenham - desde la entonación de canciones de "sonrisas y lágrimas" a las cuatro de la mañana hasta los bailes irlandeses con la policía-, estos movimientos tienen un agudo sentido de la aventura y el juego que no participa ni en la inclinación de Ghandi hacia el ensalzamiento del sufrimiento ni de su condena de la sensualidad. Por citar al activista pagano Starhawk, quien ha reinventado la antigua danza espiral para la gente contemporánea que busca aprovechar de manera cooperativa los poderes de la Tierra,

"Ghandi fue un gran hombre, pero sus ideas no siempre encajan con muchas de nosotras, particularmente las mujeres. Ghandi dijo que teníamos que aceptar y asumir el sufrimiento. Las mujeres llevan haciendo eso miles y miles de años, y no ha parado nada - excepto las vidas de muchas mujeres-. De algún modo, tampoco es ecológico. En vez de absorber la violencia, lo que necesitamos es encontrar la manera de pararla y luego transformarla, tomar esa energía y convertirla en un cambio creativo"'.

Las contadoras de cuentos y activistas ecofeministas de Kerala, India, por ejemplo, han usado poesía, canciones, representaciones pictóricas y el teatro callejero para reformular las visiones ghandianas. Así, en la marcha hasta el punto más al sur de la India en 1989, donde activistas unieron su postura en contra de la energía nuclear con una postura en contra de los arrastres pesqueros mecánicos y la demanda de un acceso equitativo al agua limpia, las mujeres insistieron en que "el agua debe ser protegida si la vida ha de ser protegida"10. Para estas mujeres, los cuerpos de las mujeres no son objetos de control, sino fuentes de fuerza. En esta emergente política de renovación, una política en la que la creatividad de nuestra especie tiene el potencial de lo que yo quiero llamar "(re)evolución", la paz y la aventura no son antitéticas sino elementos de apoyo mutuo y creativo.

Véase STARHAWK, "Power, Authority, and Mystery: Ecofeminism and Earth-based spirituality", DIAMOND, Irene, ORENSTEIN, Gloria Feman (Eds.), Reweaving the World...", op. cit., p. 79, para una discussion sobre Greenham Common.

10 Para una discusión sobre esta marcha, véase, "Break-through despite Break-up: Protect Waters! Protect Life!", Kanya Hymari March, National Fisherman's Forum, Cochin, 19 de Octubre de 1989. 
A diferencia de la política de la restructuración, una política de la renovación tiene poco interés en el control, en la normalización, o en contener lo desviado o lo bizarro, pues dicha política entiende los límites de la mente humana y de la planificación racional a escala global. La política de renovación y (re)evolución nos pide que consideremos seriamente las posibilidades de quién podemos llegar a ser en un mundo en despliegue. Esta política nos empuja más allá de nuestras identidades exclusivas - ya sean sexuales, étnicas o nacionalesy nos recuerda que nuestro ser en tanto que especie y nuestra relación con fuerzas cósmicas.

Tradicionalmente, la conciencia de las fuerzas cósmicas ha sido ignorada o desdeñada por quieres defienden un cambio revolucionario cuya atención a la lucha de clase les ha ensordecido frente a nociones de espíritu o cosmología. De igual modo, quienes ven posibilidades en el lenguaje de la evolución han extirpado cuidadosamente a la política en un modo muy similar a aquel de los técnicos que predican la restructuración. Estos esfuerzos por desplazar la política, sin embargo, están llenos de peligros. Por un lado, el problemático lenguaje de la evolución puede reforzar la arrogancia de la visión darwinista canónica que postula a los seres humanos como la expresión más alta de la creación ${ }^{11}$. Nuestra evolución en seres más cariñosos, reflexivos y ecológicamente conscientes, que anhela New Agers, es una visión valiosa, pero su realización no es en absoluto inevitable. No podemos depender de la planificación de una ley natural más elevada. Los humanos, especialmente los humanos blancos del Norte, debemos decidir conscientemente que nos vamos a reimaginar. El alcance del cambio personal e institucional necesario no puede contenerse en nociones tradicionales de cambio social revolucionario que hacen hincapié en la toma del poder estatal, ni en los planes técnicos de los actuales defensores de la restructuración, que prefieren reformar el poder estatal para facilitar los mercados globales. Dado nuestro patrón de sobreconsumo, vivir de forma ligera sobre el planeta significará necesariamente hacerlo con menos en términos materiales. A nivel de nuestras vidas personales y comunitarias, dicha experiencia bien puede experimentarse como algo alegre y espiritualmente liberadora. Pero no debe entenderse tanto como privación. Como dice Jane Benett en su alegato a favor de un "holismo fraccionado", que tolera la alteridad en la naturaleza y el orden social,

"¿Qué justificación puede dar una ética que busca expresar un mundo fraccionado y flexible para su deseo de pisar ligero?... debemos pisar ligero porque es la orientación más sabia en un mundo del que dependemos pero que no podemos controlar o comprender del todo... la existencia humana sobre el planeta es precaria, no garantizada"12.

El desafío es reparar y crear instituciones democráticas, autodependientes, frugales e igualitarias que comercien principalmente a través del trueque y de modos de intercambio cara a cara, en vez de los modos de intercambio monetarios y abstractos que entendemos por comercio. Dichas instituciones serían capaces de sobrevivir sin las conocidas seguridades del actual sistema del estado nación. Por supuesto, esta no es una tarea fácil, pero tales cambios están ocurriendo ya en lugares locales en todo el mundo. A lo largo y ancho del planeta encontramos la reinvención de redes comunitarias para el cuidado cooperativo de los niños

\footnotetext{
11 Para una crítica de la vision canónica de la evolución que refuerza la superioridad humana, véase GOULD, Stephan Jay, Wonderful Life: The Burgess Shale and the Nature of History, Norton, Nueva York, 1989.

12 BENNETT, Jane, Unthinking Faith and Enlightment, New York University Press, Nueva York, 1989, p. 158.
} 
y niñas, la seguridad pública, los jardines, las necesidades espirituales, las preparaciones funerarias, hasta los distintos esfuerzos para transformar el viajar y la aventura a través de las prácticas de turismo responsable y la invención de las redes económicas bioregionales que usan su propia moneda, y el mantenimiento o creación de mercados artesanales locales donde se intercambia información, servicios y productos autoproducidos ${ }^{13}$. Y a quienes quieran alzar el espectro de la anarquía - un falso entendimiento de lo que aquellos trabajando en la tradición colectivista del anarquismo tratan de hacer-, yo tomaría nota de la provocativa sugerencia de Thomas Kuehl, de que

"ni la naturaleza ni la humanidad están predispuestas a las operaciones del estado... mientras que la mayoría de los teóricos de la soberanía presentan a los humanos y a la naturaleza como objetos listos para la imposición de soberanía, un análisis foucaultiano resalta el trabajo que debe hacerse sobre ambas en nombre de la soberanía"14.

El trabajo de (re)evolución es sinérgico, más como las ondulaciones de espirales que se desenvuelven o de conchas, que como los procesos aditivos o lineares que típicamente asociamos con las nociones evolutivas de cambio. Debemos forjar nuevas metáforas como el baile en espiral de Starhawk para general visiones de vínculos horizontales de imprevisibilidad. En el baile, parte integral de los rituales de casi todas las culturas indígenas, los cambios rítmicos y las energías de nuestros cuerpos se vinculan con los ciclos de renovación de la Tierra. Expandiendo y enriqueciendo nuestras capacidades sensoriales, la política de (re) novación bien puede fortalecer nuestros lazos con la Tierra y con los demás, permitiéndonos experimentar en maneras positivas lo que Occidente denomina la alteridad de la naturaleza.

En el seno de esta política emergente, las polaridades tradicionales de la política moderna se reformulan. He sugerido en este capítulo que ecofeministas, verdes y bioregionalistas a menudo simpatizan con prácticas y modos de vida tradicionales. El conocimiento vernáculo es altamente respetado y valorado. A diferencia de los modernizadores ilustrados que se centran en la esperanza del futuro denigrando el pasado, la política de la renovación y la revolución crea nuevas visiones sirviéndose de la sabiduría de la tradición.

Aquí, de nuevo, hay un importante parentesco con Ghandi. Como el activista contemporáneo y editor de la revista femenina Manushi, Madhu Kishaw, ha notado, según Ghandi, "es bueno nadar en las aguas de la tradición, pero hundirse en ellas es el suicidio"15. La tradición debe usarse como guía, pero no debemos permitir que limite nuestro pensamiento a medida que respondemos a nuevas condiciones. Para los activistas de base en la India que se enfrentan a las necesidades energéticas contemporáneas, la tradición de hacer uso de las riquezas de la Tierra puede significar la invención de tecnologías que puedan convertir el compost en energía usando excrementos animales y vegetación descartada, a las cuales el

$13 \quad$ Fuentes excelentes para informes sobre dichos esfuerzos comunitarios son las revistas: The Utne Reader, Catalyst, The New Catalyst, y Rain; véase especialmente el número de Rain sobre "working communities", vol. 14, no 2, 1992; sobre las posibilidades del trueque véase SILVER, Michell, "The Ultimate Barter" en Mother Earth News, Agosto/Septiembre, 1993, sobre enfoques económicos bioregionales véase LOWRY, Susan Meeker, Economics as if the Earth Really Mattered, New Society Publishers, Philadelphia, 1988.

14 KUEHL, Thomas, "The Nature of the State: An Ecological Rereading of Sovereignty and Territory" en KUEHL, Thomas, Reimagining the Nation, Open University Press, Londres, de próxima publicación.

15 KISHWAR, Madhu, Ghandi and Women, Manushi Prakashan, Nueva Delhi, 1986, p. 2. 
desarrollo ha definido como basura. En efecto, para muchos activistas dentro del movimiento por una tecnología adecuada, la biomasa producida localmente es central para la visión de sociedades centradas en la aldea.

En el seno de la política de la renovación, la conciencia de la gente y las prácticas que nos precedieron resultan necesaria para determinar las repercusiones de nuestras actividades y tecnologías presentes. Resistiendo nuestro moderno lenguaje de control, e invocando el respeto por la fertilidad de la tierra, nuestros cuerpos, y las comunidades que nutren nuestros corazones y nuestras mentes, ecofeministas y verdes buscan sostener tanto a la Tierra viviente como a todas sus criaturas únicas. Este compromiso con la diversidad no es un ideal abstracto o un sustituto fácil para la verdad eterna. La diversidad es básica para el bienestar ecosistémico. Oponiendo a la visión homogeneizadora y elitista del control tecnológico un entendimiento más complejo de la diversidad y las prácticas de los pueblos indígenas que honran la continuidad y la sacralidad de la vida, ecofeministas y verdes luchan por preservar y transformar la comunidad y la democracia en el mundo moderno.

En vez de lamentarse por el fin de la naturaleza, nosotros, humanos, mujeres y hombres, debemos (re)evolucionar en seres que prefieren nuestros jardines locales. Debemos resistir la llamada de la tradición occidental de llevar la ilustración a todo el planeta. Aunque parezca paradójico, el respeto a la fertilidad -en todas sus diversas formas: vegetal, social e imaginativa- puede ser el medio más prudente, moral, y políticamente efectivo para sobrevivir en el siglo veintiuno. La triste historia del lenguaje del control nos dice que la difícil, aunque esencial tarea de crear comunidades que honren los muchos asombros de la fertilidad, es la tarea primaria que tenemos ante nosotros. La Tierra viva Ilama.

\section{Bibliografía}

BAGBY, Rachel, "Daughters of Growing Things" en DIAMOND, Irene, ORENSTEIN, Gloria Feman (eds.), Reweaving the World: The Emergence of Ecofeminism, Sierra Club, San Francisco, 1990.

BENNETT, Jane, Unthinking Faith and Enlightment, New York University Press, Nueva York, 1989.

GARB, Jaakov Jerome, "Perspective or escape? Ecofeminist Musings on Contemporary Earth Imaginery" en DIAMOND, Irene, ORENSTEIN, Gloria Feman (eds.), Reweaving the World: The Emergence of Ecofeminism, Sierra Club, San Francisco, 1990.

GOMES, Teresa Santa Clara, "A Feminist Utopia" en Terra Femina.

GOULD, Stephan Jay, Wonderful Life: The Burgess Shale and the Nature of History, Norton, Nueva York, 1989.

GURU, Gopal, "Latmi Mukti" en Economic and Political Weekly, Julio, 1992.

KISHWAR, Madhu, Ghandi and Women, Manushi Prakashan, Nueva Delhi, 1986,

KUEHL, Thomas, "The Nature of the State: An Ecological Rereading of Sovereignty and Territory" en KUEHL, Thomas, Reimagining the Nation, Open University Press, Londres, de próxima publicación.

LEVINE, Murray, Love Canal: My Story, State University Press of New York, Albany, 1982;

LOWRY, Susan Meeker, Economics as if the Earth Really Mattered, New Society Publishers, Philadelphia, 1988.

MAATHAI, Wangari, The Greenbeld Movement, Environmental Liason Centre International, Nairobi, 1988;

OMVEDT, Gail, "Latmi Mukti" en Hitavada, 27 de Diciembre, 1992. 
OMVEDT, Gail, The Awakening of Women's Power: The Rural Women's Movement in India, manuscrito no publicado, 1992.

PATKAR, Medha [Entrevista con] en India International Centre Quarterly 1990, vol. 19, n 1-2, 1992, ps. 273-99.

SEN, Ilina, (ed.), A Space Within the Struggle: Women's Participation in People's Movements, Kali for Women, Nueva Deli, 1990.

SILVER, Michell, "The Ultimate Barter" en Mother Earth News, Agosto/Septiembre, 1993.

STARHAWK, "Power, Authority, and Mystery: Ecofeminism and Earth-based spirituality" en DIAMOND, Irene, ORENSTEIN, Gloria Feman (eds.), Reweaving the World: The Emergence of Ecofeminism, Sierra Club, San Francisco, 1990.

VISVANATAN, Shiv, "Mrs. Brudtland's disenchanted Cosmos" en Alternatives, Social Transformation and Humane Governance, vol. 16, no 3, 1991, ps. 377-84.

VVAA, "Global Management" en The Ecologist, vol. 22, n० 3, 1992.

VVAA, "Whose Common Future?" en The Ecologist, vol. 22, n 3, 1992.

VVAA, "Working communities" en Rain, vol. 14, n 2, 1992. 


\section{RELACIONES INTERNACIONALES}

Revista académica cuatrimestral de publicación electrónica Grupo de Estudios de Relaciones Internacionales (GERI) Universidad Autónoma de Madrid, España

www.relacionesinternacionales.info

ISSN 1699 - 3950

ff facebook.com/RelacionesInternacionales

twitter.com/RRInternacional 